\title{
Bannayan-Riley-Ruvalcaba Syndrome: MRI Neuroimaging Features in a Series of 7 Patients
}

\author{
R. Bhargava, K.J. Au Yong, and N. Leonard
}

\begin{abstract}
SUMMARY: Bannayan-Riley-Ruvalcaba syndrome is a congenital disorder characterized by macrocephaly, intestinal polyposis, lipomas, and pigmented macules of the penis. There is limited published radiologic literature on the syndrome. The purpose of this study was to review the brain MR imaging findings in Bannayan-Riley-Ruvalcaba syndrome as well as to compare and contrast the findings with other brain disorders that also have brain cysts and white matter lesions. All brain MR imaging studies were reviewed in patients with a diagnosis of Bannayan-Riley-Ruvalcaba syndrome from our hospital. All 7 patients were evaluated with brain MR imaging. MR imaging results showed white matter cysts in the parietal lobe (7/7), frontal lobe (3/7), and temporal lobe (1/7). These were predominantly surrounded by white matter T2 hyperintensities associated with macrocephaly. Cystic lesions on MR imaging in Bannayan-Riley-Ruvalcaba syndrome are prevalent, and knowledge of this differential diagnosis can allow the radiologist to suggest a diagnosis of this condition in a child with macrocephaly.
\end{abstract}

ABBREVATIONS: BRRS = Bannayan-Riley-Ruvalcaba syndrome; $C S=$ Cowden syndrome; $L D D=$ Lhermitte-Duclos disease; PTEN $=$ phosphatase and tensin homolog; VR = Virchow-Robin

B annayan-Riley-Ruvalcaba syndrome (BRRS) classically presents with macrocephaly, subcutaneous and visceral lipomata, hemangiomata, hamartomatous intestinal polyps, and pigmented macules involving the genitalia.

This autosomal dominant disorder is linked to germline mutations of the phosphatase and tensin homolog gene (PTEN), a tumor suppressor gene localized to 10q23 that has a significant role in the molecular pathway of cellular proliferation, migration, and apoptosis. In 1996, it was recognized that BRRS shared features with Cowden syndrome (CS), another autosomal dominant condition with multiple hamartomas. ${ }^{1}$

An estimated $65 \%$ of patients with a clinical diagnosis of BRRS have a detectable PTEN mutation. When BRRS is not caused by mutations or deletions of the PTEN gene, the cause of the condition is unknown.

Other features would include high birth weight, developmental delay, and intellectual disability (50\%); a myopathic process in

Received April 14, 2013; accepted after revision May 27.

From the Departments of Radiology and Diagnostic Imaging (R.B.), and Medical Genetics (N.L.), University of Alberta Hospital, Edmonton, Alberta, Canada; and Department of Radiology and Diagnostic Imaging (K.J.A.), McMaster University Medical Centre, McMaster Children's Hospital, Hamilton, Ontario, Canada.

Please address correspondence to Kong Jung Au Yong, MB ChB, MRCS, FRCR, MBA, Department of Radiology and Diagnostic Imaging, McMaster University Medical

Centre, McMaster Children's Hospital, 1280 Main St West, Hamilton, ON L8S 4L8,

Canada; e-mail: kong.auyong@gmail.com

http://dx.doi.org/10.3174/ajnr.A3680

proximal muscles (60\%); and joint hyperextensibility, pectus excavatum, and scoliosis (50\%). ${ }^{2}$ Abnormal facial features include frontal bossing, hypertelorism, down-slanting palpebral fissures, depressed nasal bridge, strabismus, epicanthus inversus, small beaked nose, long philtrum, thin upper lip, broad mouth, and relative micrognathia. ${ }^{3}$

There are no agreed international criteria for the diagnosis of BRRS. The criteria suggested by Marsh et $\mathrm{al}^{4}$ should include at least the following 4 features: macrocephaly, lipomatosis, hemangiomas, and speckled penis in boys. The other criteria suggested by Parisi et $\mathrm{al}^{5}$ should include at least 2 of the following characteristics: macrocephaly, hamartomas (including at least 1 lipoma, hemangioma, or intestinal polyp), and penile macules in boys. Although cancer was initially not believed to be a component of the syndrome, people with BRRS and a PTEN mutation are currently thought to have the same cancer risks as those with CS. ${ }^{4}$

PTEN, on 10q23.3, encodes a major lipid phosphatase that signals down the phosphoinositol-3-kinase/Akt pathway and effects G1 cell cycle arrest and apoptosis. Germline PTEN mutations have been found to occur in $80 \%$ of classic CS, $60 \%$ of BRRS, up to $20 \%$ of Proteus syndrome, and approximately $50 \%$ of a Proteuslike syndrome. ${ }^{6}$ PTEN hamartoma tumor syndrome now encompasses 4 major clinically distinct syndromes associated with germline mutations in the tumor suppressor PTEN. ${ }^{7} \mathrm{CS}$ is rarely diagnosed before adolescence; BRRS is generally reported with a childhood onset, often with delayed motor and intellectual devel- 
Summary of MRI and clinical characteristic of 7 patients diagnosed with BRRS

\begin{tabular}{|c|c|c|c|c|c|c|c|c|}
\hline $\begin{array}{l}\text { Patient } \\
\text { No. }\end{array}$ & Sex & $\begin{array}{l}\text { Age at } \\
\text { MRI (y) }\end{array}$ & Macrocephaly & $\begin{array}{l}\text { Mutation } \\
\text { PTEN }\end{array}$ & Development & Physical Features & WM Cyst & $\begin{array}{c}\text { Cyst } \\
\text { Location }\end{array}$ \\
\hline 1 & M & 4 & Yes & Yes & Autistic & Abdominal hemangioma & Yes & $f, p, t$ \\
\hline 2 & M & 0.65 & Yes & Yes & Delay & $\begin{array}{l}\text { Café au lait spot, thyroid nodules, testicular } \\
\text { hamartomas, rectal and gastric polyps }\end{array}$ & Yes & $\mathrm{p}$ \\
\hline 3 & M & 2.7 & Yes & No protein & Autistic & Motor speech disorder & Yes & $f, p$ \\
\hline 4 & M & 3.5 & 90th centile & No protein & Delay & Café au lait spot & Yes & $\mathrm{p}$ \\
\hline 5 & M & 2.6 & Yes & No protein & Delay & Moles, thyroid nodules, intestinal polyps & Yes & p \\
\hline 6 & M & 7.7 & Yes & No protein & Delay & Moles, thyroid nodules, intestinal polyps & Yes & p \\
\hline 7 & $M$ & 7.7 & Yes & Yes & Delay & $\begin{array}{l}\text { Pigmentation of the glans penis, multiple thyroid } \\
\text { nodules, and carcinoma }\end{array}$ & Yes & $f, p$ \\
\hline
\end{tabular}

Note:- $f$ indicates frontal; $p$, parietal; t, temporal.

opment. Most studies have failed to demonstrate a consistent genotype-phenotype relationship between CS and BRRS, but recently, studies have suggested that the unique features in the 2 syndromes may actually represent age-related penetrance of the same condition. ${ }^{8}$ People with CS and BRRS within the same family (CS/BRRS overlap families) have been reported. ${ }^{9}$ Therefore, early diagnosis of BRRS at a young age may allow patients to be monitored for the onset of malignant disease.

With increased recognition of the syndrome, neuroimaging signs such as AVMs and increased perivascular spaces have been recently described. ${ }^{10,11}$ Therefore, it is important for radiologists to be aware of the neuroimaging abnormalities found in patients with BRRS to allow accurate diagnosis and provide preventive care. We have reviewed the neuroimaging features of 7 patients with BRRS.

\section{MATERIALS AND METHODS}

Local Human Research Ethics board approval was obtained to review the clinical and imaging records of patients with BRRS without patient consent. We searched the genetic disorder data base at our institution for all cases of BRRS diagnosed since 1994. All available MR imaging studies and clinical records were reviewed in patients with a diagnosis of BRRS.

All patients had a review by geneticists and were tested for the PTEN gene mutation. To our knowledge, no diagnostic criteria for BRRS have been set. We based our diagnosis heavily on the presence of cardinal features of macrocephaly, developmental delay/autism, characteristic triangular facial feature, prominent forehead, malar hypoplasia, down-slanting palpebral fissures, myopathic features, and pigmented macules of the glans penis associated with the PTEN mutation.

All MR imaging was performed in routine clinical care by use of 1.5T scanning. Standard 3D MPRAGE T1-weighted, fast spinecho T2-weighted, and FLAIR images were available for all patients. The MR imaging results in each patient were reviewed separately and then jointly by 2 pediatric neuroradiologists. Systematic chart review was used to document the clinical details, mode of presentation, patient demographics, clinical features, and MR imaging brain features.

\section{RESULTS}

Since 1994, a total of 7 patients (all boys) have been diagnosed with BRRS at the University of Alberta Hospital (Edmonton, Alberta, Canada), and all 7 were evaluated with brain MR imaging. The accompanying table summarizes the neuroimaging and clinical findings of these 7 patients.

\section{Patient Demographics}

The age at presentation ranged from 8 months -7.7 years. Patients 5 and 6 were siblings.

\section{PTEN Gene}

Three patients had the PTEN mutation and 4 had absence of the PTEN protein, indicating an abnormality of PTEN.

\section{Clinical Presentation}

All 7 of our patients presented with developmental delay or autism spectrum disorder. Skin pigmentation, nevi, or café au lait spots were seen in 5 of 7 patients. Three patients had intestinal polyps, and 4 had thyroid nodules. One patient had testicular hamartomas. Thyroid carcinoma developed in another patient at age 16 years.

The distinctive finding of penile macules in boys (seen in $67 \%$ of patients with BRRS) is a major criterion in BRRS but may not appear until mid-childhood and was only seen in 2 of our patients at presentation. PTEN mutations can have many different phenotypes; therefore, our diagnosis of BRRS was guided more specifically by the facial features; pigmented macules of the glans; myopathy; and subsequent determination of intestinal hamartomatous polyps, which separates them from Cowden and Proteus. The features of Cowden and Proteus, which are also associated with PTEN mutations, were not seen in our patients.

\section{MR Imaging Brain Features}

All of our 7 patients had MR imaging of the brain, as shown in the accompanying figures (Fig 1). The imaging findings include CSF signal intensity (hyperintense on T2 and hypointense on FLAIR) and white matter cysts in the parietal lobes (7/7), frontal lobes $(3 / 7)$, and temporal lobes (1/7). These were associated with white matter T2 hyperintensities predominantly around the cysts (7/7). Additional findings in 1 patient included a cavum velum interpositum arachnoid cyst. All but 1 patient (above the 90th centile) had extreme macrocephaly.

\section{DISCUSSION}

BRRS has been shown to affect the skin and central nervous system. However, descriptions of neuroimaging studies are uncommon in the literature. To our knowledge, no similar case series has specifically studied the neuroimaging findings of BRRS.

In a recent study of 181 patients with pathogenic germline PTEN mutations, 152 (94.4\%) had macrocephaly as defined by a 

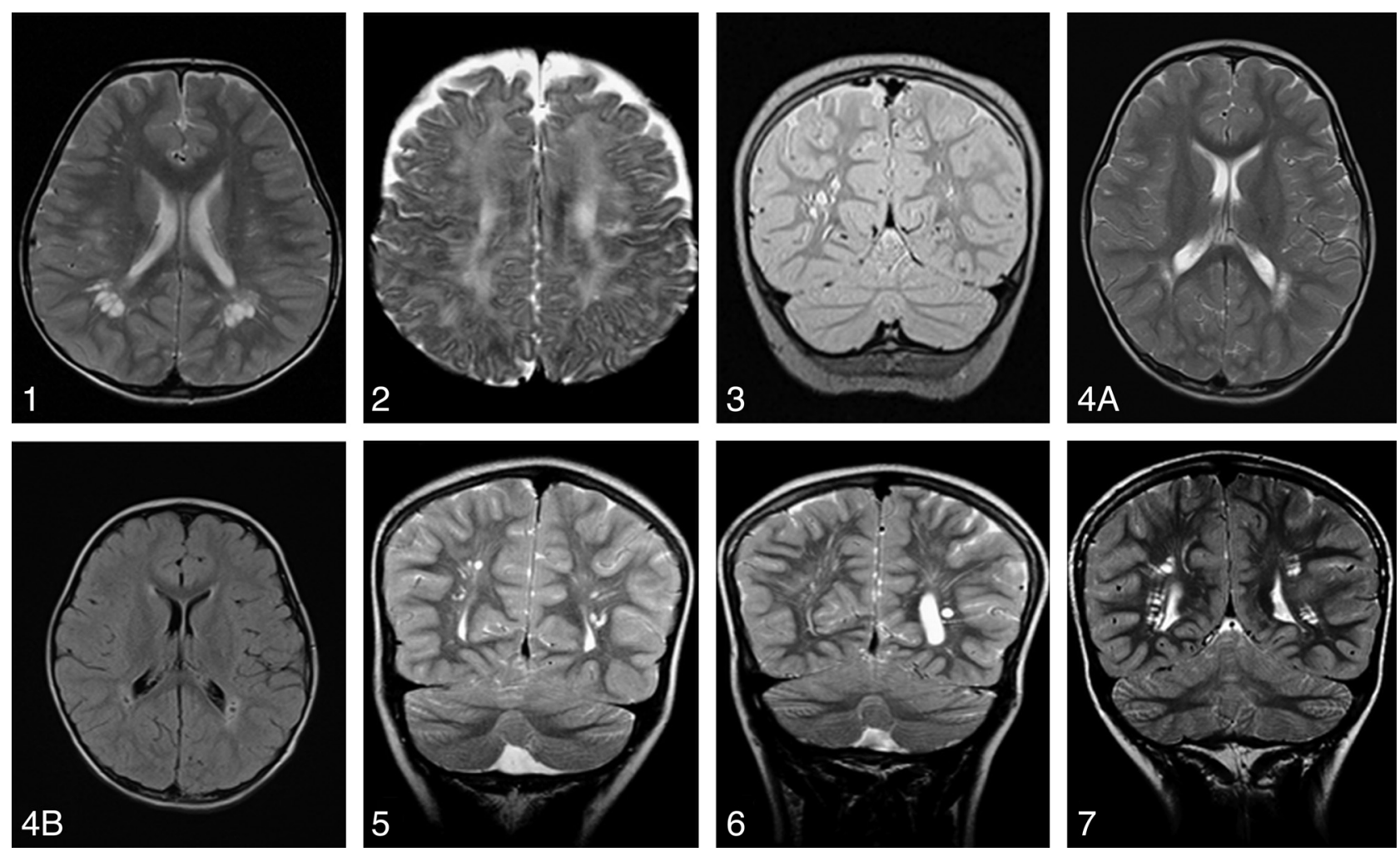

FIG 1. 1, Axial T2 image in patient 1 demonstrates bilateral cystic spaces in the biparietal periventricular white matter. 2, Axial T2 image in patient 2 demonstrating small cystic spaces in the parietal white matter associated with increased T2 signal of the periventricular white matter. 3, Coronal T2 image in patient 3 demonstrates cystic spaces in the periventricular white matter of the parietal lobes. 4A, Axial T2 image in patient 4 demonstrates bilateral small parietal periventricular cyst associated with white matter T2 hyperintensities predominantly around the cysts. $4 B$, An axial FLAIR image in patient 4 demonstrates pericyst hyperintensities and suppression of the VR space fluid. 5, Coronal T2 image of patient 5 demonstrates a well-defined small periventricular cyst in the parietal region. 6, Coronal T2 image in patient 6 demonstrates a well-defined small periventricular cyst in the left parietal region. 7, Coronal T2 image in patient 7 demonstrates small periventricular cysts in the biparietal region.

head circumference of 2 SD over the mean. There was no significant difference in age or sex. Macrocephaly was observed in all but one of our patients. Studies on mice specimens with PTEN mutations have shown megalencephaly. ${ }^{12}$ The size of the ventricles and the extra-axial spaces in all of our patients were within normal limits in keeping with megalencephaly.

Megalencephaly in BRRS is often associated with overgrowth features but can be distinguished from other causes of megalencephaly with overgrowth such as Soto syndrome, neurofibromatosis type 1, Simpson-Golabi-Behmel, Fragile X, Weaver, and macrocephaly-cutis marmorata telangiectatica congenita by use of a combination of dysmorphic features, associated malformations, family history, and specific growth patterns. ${ }^{13}$

All of our patients were referred for neuroimaging because of developmental delay or autism. Cognitive impairment is reported to be between $20 \%-50 \%$ in patients with BRRS, which is higher than in those with CS at $10 \% .{ }^{14}$ In a study of 18 patients with autism with a head circumference range from 2.5-8.0 SD above the mean and with autism spectrum disorder, 3 boys (17\%) carried germline PTEN mutations. Two of 7 patients in our study have autistic spectrum disorder. ${ }^{15}$ A total of 24 patients with PTEN mutation, autism, macrocephaly, and some clinical findings described in PTEN syndromes have been reported in the literature. ${ }^{16}$ Approximately $25 \%$ of patients with BRRS have seizures. ${ }^{17}$ Cortical malformation is considered rare in BRRS, with only 3 cases associated with PTEN mutation reported in the liter- ature. ${ }^{18}$ None of our patients demonstrated any cortical malformation or midline defects. A small cavum velum interpositum arachnoid cyst was found in 1 patient.

The PTEN gene, a tumor suppressor, contains a phosphatase domain, and its protein product has phosphatase activity. Mutations of this gene have been detected in glioblastoma cell lines and tumors. In a large series, 74\% (25/34) of glioblastomas demonstrated loss of heterozygosity at the PTEN locus, and 60\% (15/25) of these cases contained somatic PTEN mutations of both alleles, implicating the PTEN gene in tumor development. ${ }^{19}$

Published case reports have described rare cases of meningiomas, frontal vascular hamartoma, intracranial lipomatous hamartoma, and malignant intracranial human chorionic gonadotropin-secreting tumor in patients with BRRS. ${ }^{14,20}$

The PTEN/PI3K pathway governs normal vascular development and tumor angiogenesis. ${ }^{21}$ High-flow vascular malformations, ${ }^{1}$ including dural AVM, ${ }^{10}$ hemangiomata, and vertebral angiomas, ${ }^{22}$ may exist in patients with BRRS.

In a study of 26 patients with PTEN mutation ${ }^{23}$, vascular anomalies were found in 14 (54\%) patients. Intracranial developmental venous anomalies were found in $8(89 \%)$ of 9 patients who had brain MR imaging with contrast. ${ }^{23}$ High frequency of vascular malformations was noted in a study of 20 patients with CS, where 5 patients had venous angiomas (3 associated with Lhermitte-Duclos disease [LDD]) and 2 patients had cavernous angi- 
omas ( 1 associated with LDD) with a significant proportion related to LDD. ${ }^{14}$

No vascular anomalies were found in our 7 patients, but this probably is an underestimate because none of our patients received contrast material. More recently, congenitally enlarged VR spaces have been described in a case of BRRS. ${ }^{11}$

Our 7 patients demonstrated cystic dilation of the VR spaces at the white matter of the centrum semiovale and parietal region. There were round, oval, or curvilinear well-defined cystic lesions with smooth margins and isointense to CSF. On FLAIR imaging, there was suppression of the VR space fluid. One follow-up MR imaging study was available for comparison, and the cystic dilation of the VR spaces appeared nonprogressive. Lok et $\mathrm{al}^{14}$ reported cystic dilation of the VR spaces in 3 (15\%) of 20 patients (ages 34-56 years) with CS. Merks et $\mathrm{al}^{24}$ described a mother and 3 sons with CS/BRRS and variable phenotype: 1 family member having macrocephaly, normal intelligence, and minimal pigmentation abnormalities; another member with macrocephaly with developmental delay; another with macrocephaly, delay and lipoma; and the last family member having hemimegalencephaly, Jadassohn naevus sebaceous, and neonatal demise. Two of the 3 sons have periventricular cyst or dilated perivascular spaces.

It is unknown if the cystic VR space dilations in our patients constitute a physiopathologic marker, but the extent encountered in our patients has not been described in unaffected children; therefore, a causal relationship with the presenting signs is probable.

The cystic dilation of VR spaces discussed above is nonspecific and does not distinguish it from other diseases that cause white matter brain cysts such as Soto syndrome, Lowe syndrome, mucopolysaccharidosis, and Van der Knaap syndrome; however, ancillary imaging findings allow for diagnosis of these conditions. Soto syndrome is frequently associated with large ventricles, increased extracerebral fluid, and midline anomalies. ${ }^{25}$ In Lowe syndrome, hyperintensities on T2-weighted images and periventricular cystic lesions have been described; however, the brain size is usually below normal and is associated with white matter gliosis (increased myo-inositol peak) mainly in the centrum semiovale, atrophy, ventricular enlargement, and thinning of the corpus callosum. ${ }^{26}$ The disease is also associated with bilateral congenital cataracts, glaucoma, hypotonia, severe psychomotor retardation, seizures, and disturbances of renal tubular function. ${ }^{27}$ Neuroimaging findings such as hydrocephalus, white matter lesions, brain atrophy, MR spectroscopy changes (a low NAA/Cr and elevated mIns/Cr), and clinical phenotype can easily distinguish mucopolysaccharidosis from BRRS. Thickening of the diploic space, sellar abnormalities, or vertebral body changes seen on head MR imaging can also allow for differentiation of a mucopolysaccharidosis from BRRS. Finally, Van der Knaap syndrome is associated with extensive white matter changes, and the cysts are usually subcortical in position, sparing the deeper structures. ${ }^{28}$

Our small retrospective study had some limitations. This frequency of cerebral abnormalities in patients with BRRS was surely an underestimate, as serial neuroimaging or contrast imaging has not been performed. Because our study was small, we found it difficult to draw statistically significant conclusions. All of the patients in our study had been referred for a pediatric medical opinion because of concerns about early motor development or learning difficulties, and this ascertainment bias may have led to an overestimate of the risk of learning difficulties in children diagnosed with BRRS.

\section{CONCLUSIONS}

Although a rare syndrome, the cystic lesions seen on brain imaging findings in BRRS are quite prevalent. Future studies of larger populations and long-term follow-up are needed to confirm our preliminary MR imaging findings. Knowledge of the differential diagnosis of macrocephaly and brain cystic lesions in the setting of a white matter disorder can allow the radiologist to suggest a diagnosis of BRRS on brain MR imaging. Macrocephaly, together with cystic VR spaces on MR imaging, may be a useful diagnostic pointer, particularly when deciding which patients to test for PTEN mutation.

Disclosures: Norma Leonard-RELATED: Payment for Writing or Reviewing the Manuscript: University of Alberta (clarify).

\section{REFERENCES}

1. Fargnoli MC, Orlow SJ, Semel-Concepcion J, et al. Clinicopathologic findings in the Bannayan-Riley-Ruvalcaba syndrome. Arch Dermatol 1996;132:1214-18

2. Zbuk KM, Eng C. Cancer phenomics: RET and PTEN as illustrative models. Nat Rev Cancer 2007;7:35-45

3. Hendriks YM, Verhallen JT, van der Smagt JJ, et al. Bannayan-RileyRuvalcaba syndrome: further delineation of the phenotype and management of PTEN mutation-positive cases. Fam Cancer 2003;2:79-85

4. Marsh DJ, Kum JB, Lunetta KL, et al. PTEN mutation spectrum and genotype-phenotype correlations in Bannayan-Riley-Ruvalcaba syndrome suggest a single entity with Cowden syndrome. Hum Mol Genet 1999;8:1461-72

5. Parisi MA, Dinulos MB, Leppig KA, et al. The spectrum and evolution of phenotypic findings in PTEN mutation positive cases of BannayanRiley-Ruvalcaba syndrome. J Med Genet 2001;38:52-58

6. Eng C. PTEN: one gene, many syndromes. Hum Mutat 2003;22:183-98

7. Hobert JA, Eng C. PTEN hamartoma tumor syndrome: an overview. Genet Med 2009;11:687-94

8. Lachlan KL, Lucassen AM, Bunyan D, et al. Cowden syndrome and Bannayan Riley Ruvalcaba syndrome represent one condition with variable expression and age-related penetrance: results of a clinical study of PTEN mutation carriers. J Med Genet 2007;44:579-85

9. Wanner M, Celebi JT, Peacocke M. Identification of a PTEN mutation in a family with Cowden syndrome and Bannayan-Zonana syndrome. J Am Acad Dermatol 2001;44:183-87

10. Srinivasa RN, Burrows PE. Dural arteriovenous malformation in a child with Bannayan-Riley-Ruvalcaba syndrome. AJNR Am J Neuroradiol 2006;27:1927-29

11. Nebgen S, Zerres K, Deutz U, et al. Bannayan-Riley-Ruvalcaba-syndrome-an unusual differential diagnosis of dilatated VirchowRobin spaces. Neuropediatrics 2012;43 - PS13_06

12. Mester JL, Tilot AK, Rybicki LA, et al. Analysis of prevalence and degree of macrocephaly in patients with germline PTEN mutations and of brain weight in PTEN knock-in murine model. Eur J Hum Genet 2011;19:763-68

13. Olney AH. Macrocephaly syndromes. Semin Pediatr Neurol 2007; 14:128-35

14. Lok C, Viseux V, Avril MF, et al. Brain magnetic resonance imaging in patients with Cowden syndrome. Medicine (Baltimore) 2005;84:129-36

15. Butler MG, Dasouki MJ, Zhou XP, et al. Subset of individuals with autism spectrum disorders and extreme macrocephaly associated with germline PTEN tumour suppressor gene mutations. J Med Genet 2005;42:318-21 
16. Conti S, Condò M, Posar A, et al. Phosphatase and tensin homolog (PTEN) gene mutations and autism: literature review and a case report of a patient with Cowden syndrome, autistic disorder, and epilepsy. J Child Neurol 2012;27:392-97

17. DiLiberti JH, D’Agostino AN, Ruvalcaba RH, et al. A new lipid storage myopathy observed in individuals with the Ruvalcaba-MyhreSmith syndrome. Am J Med Genet 1984;18:163-67

18. O’Rourke DJ, Twomey E, Lynch SA, et al. Cortical dysplasia associated with the PTEN mutation in Bannayan Riley Ruvalcaba syndrome: a rare finding. Clin Dysmorphol 2012;21:91-92

19. Wang SI, Puc J, Li J, et al. Somatic mutations of PTEN in glioblastoma multiforme. Cancer Res 1997;57:4183-86

20. Faisal Ahmed S, Marsh DJ, Weremowicz S, et al. Balanced translocation of $10 \mathrm{q}$ and $13 \mathrm{q}$, including the PTEN gene, in a boy with a human chorionic gonadotropin-secreting tumor and the Bannayan-RileyRuvalcaba syndrome. J Clin Endocrinol Metab 1999;84:4665-70

21. Hamada K, Sasaki T, Koni PA, et al. The PTEN/PI3K pathway governs normal vascular development and tumor angiogenesis. Genes Dev 2005;19:2054-65
22. Pancaro C, Miller T, Dingeman RS. Anesthetic management of a child with Bannayan-Riley-Ruvalcaba syndrome. Anesth Analg 2008;106:1928-29

23. Tan WH, Baris HN, Burrows PE, et al. The spectrum of vascular anomalies in patients with PTEN mutations: implications for diagnosis and management. J Med Genet 2007;44:594-602

24. Merks JH, de Vries LS, Zhou XP, et al. PTEN hamartoma tumour syndrome: variability of an entity. J Med Genet 2003;40:e111

25. Schaefer GB, Bodensteiner JB, Buehler BA, et al. The neuroimaging findings in Sotos syndrome. Am J Med Genet 1997;68:462-65

26. Carvalho-Neto A, Ono SE, Cardoso Gde M, et al. Oculocerebrorenal syndrome of Lowe: Magnetic resonance imaging findings in the first six years of life. Arq Neuropsiquiatr 2009;67:305-07

27. Demmer LA, Wippold FJ 2nd, Dowton SB. Periventricular white matter cystic lesions in Lowe (oculocerebrorenal) syndrome. A new MR finding. Pediatr Radiol 1992;22:76-77

28. Batla A, Pandey S, Nehru R. Megalencephalic leukoencephalopathy with subcortical cysts: A report of four cases. J Pediatr Neurosci 2011;6:74-77 